\title{
Modified Proca Theory in Arbitrary and Two Dimensions
}

\author{
A. K. Rao ${ }^{(a)}$, R. P. Malik ${ }^{(a, b)}$ \\ (a) Physics Department, Institute of Science, \\ Banaras Hindu University, Varanasi - 221 005, (U.P.), India \\ (b) DST Centre for Interdisciplinary Mathematical Sciences, \\ Institute of Science, Banaras Hindu University, Varanasi - 221 005, India \\ e-mails: amit.akrao@gmail.com; rpmalik1995@gmail.com
}

\begin{abstract}
We demonstrate that the standard Stückelberg-modified Proca theory (i.e. a massive Abelian 1-form theory) respects the classical gauge and corresponding quantum (anti-)BRST symmetry transformations in any arbitrary dimension of spacetime within the framework of Becchi-Rouet-Stora-Tyutin (BRST) formalism. We further show that the Stückelberg formalism gets modified in the two $(1+1)$-dimensions of spacetime due to a couple of discrete duality symmetries in the theory which turn out to be responsible for the existence of the nilpotent (anti-)co-BRST symmetry transformations corresponding to the nilpotent (anti-)BRST symmetry transformations of our theory. These nilpotent symmetries exist together in the modified version of the two (1+1)-dimensional (2D) Proca theory. We provide the mathematical basis for the modification of the Stückelberg technique, the existence of the discrete duality as well as the continuous (anti-)co-BRST symmetry transformations in the 2D modified version of Proca theory.
\end{abstract}

PACS numbers: 03.70.+k; 11.30.-j; 02.40.-k.

Keywords: Modified 2D Proca theory; (anti-)BRST symmetries; (anti-)co-BRST symmetries; discrete duality symmetry transformations; a tractable model for the Hodge theory 


\section{Introduction}

The celebrated Proca theory (i.e., a massive Abelian 1-form theory) is the generalization of the Maxwell (i.e., a massless Abelian 1-form) gauge theory. Whereas the latter corresponds to a physical photon with two physical degrees of freedom, the former describes a massive vector boson with three physical degrees of freedom in the physical four $(3+1)$-dimensions of spacetime. The key signature of the Maxwell theory is the existence of the first-class constraints on it (see, e.g., $[1,2]$ ). On the contrary, the Proca theory is endowed with the second-class constraints in the terminology of Dirac's prescription for the classification scheme of constraints [1, 2]. The well-known Stückelberg technique (see, e.g., [3] for details) converts the second-class constraints of the Proca theory into the first-class constraints thereby restoring the beautiful gauge symmetry for even the massive Abelian 1-form theory where the gauge invariance and mass co-exist together in a beautiful fashion. This statement is true in any arbitrary dimension of spacetime and it is valid for even the higher $p$-form $(p=2,3 \ldots)$ gauge theories. For instance, we have exploited this technique in the context of massive Abelian 2-form gauge theory $[4,5]$ where the mass and gauge symmetry co-exist together (due to the application of the Stückelberg formalism).

The central purpose of our present endeavor is to show that there is a mathematicallybacked precise modification of the Stückelberg technique for the $p$-form $(p=1,2,3 \ldots)$ massive gauge theories in $D=2 p$ dimensions of spacetime as these theories turn out to be the massive models of Hodge theory within the framework of Becchi-Rouet-Stora-Tyutin (BRST) formalism. To corroborate this claim, in our present endeavor, we take up the 2D Stückelberg-modified Proca theory (i.e. a massive Abelian 1-form theory) to establish that there is a precise modification of the Stückelberg technique [cf. Eq. (15) below] which leads to the existence of the (anti-)co-BRST symmetries corresponding to the nilpotent (anti-) BRST symmetries (that exist in any arbitrary dimension of spacetime) because there is a set of discrete duality symmetry transformations [cf. Eqs. (23), (29)] in our 2D theory. We also show that the physical state of our theory is annihilated by the first-class constraints as well as their dual versions when we choose the physical state to be the harmonic state (of the Hodge decomposed state in the quantum Hilbert space) that is required to be annihilated by the conserved (anti-)BRST and (anti-)co-BRST charges of our modified 2D Proca theory which has been proven to be an interesting model for the Hodge theory [6, 7]. We have also demonstrated that the discrete duality symmetry transformations [see, Eq. (29) below] connect the (anti-)BRST charges with the (anti-)co-BRST charges and vice-versa. Hence, the restrictions imposed by these conserved and nilpotent charges on the physical state are also intimately connected due to the duality transformations (29). These restrictions and their connections are true at the tree-level as well as any arbitrary loop-level diagrams in the perturbative computations for a given physical process.

The theoretical contents of our present endeavor are organized as follows. First of all, to set the notations and convention, we begin with the general form of the Proca theory in the next section and discuss the (anti-)BRST symmetries in any arbitrary dimension of spacetime for the Stückelberg-modified version of this theory. The third section is devoted to the modification of the standard Stückelberg formalism in 2D for the massive Abelian 1-form (i.e. Proca) theory where we provide the mathematical basis for the existence as well as validity of this modification. The subject matter of the fourth section deals with the 
existence of the (anti-)BRST and (anti-)co-BRST symmetries together and their intimate relationships due to the existence of the discrete duality symmetry [cf. Eq. (29)] for the modified version of 2D Proca theory. Finally, in the last section, we summarize our key results and point out a few future directions for further investigations in the context of higher $p$-form $(p=2,3 \ldots)$ theories.

\section{Preliminaries: (Anti-)BRST Symmetries}

We begin with the following Lagrangian density $\left[\mathcal{L}_{(P)}\right]$ for the massive Abelian 1-form $\left(A^{(1)}=d x^{\mu} A_{\mu}\right)$ vector-boson in any arbitrary dimension of spacetime (see, e.g. [3])

$$
\mathcal{L}_{(P)}=-\frac{1}{4} F_{\mu \nu} F^{\mu \nu}+\frac{m^{2}}{2} A_{\mu} A^{\mu}
$$

where the field strength tensor $F_{\mu \nu}=\partial_{\mu} A_{\nu}-\partial_{\nu} A_{\mu}$ (with $\mu, \nu \ldots=0,1,2 \ldots D-1$ ) has been derived from the 2-form $F^{(2)}=d A^{(1)}=\frac{1}{2 !}\left(d x^{\mu} \wedge d x^{\nu}\right) F_{\mu \nu}$ where $d=d x^{\mu} \partial_{\mu}\left(\right.$ with $\left.d^{2}=0\right)$ is the exterior derivative of the differential geometry [8-10] and $m$ is the rest mass of the vector boson. It can be readily checked that under the following standard Stückelberg technique, the vector bosonic field $A_{\mu}$ is modified in the following manner [3]

$$
A_{\mu} \longrightarrow A_{\mu} \mp \frac{1}{m} \partial_{\mu} \phi
$$

where $\phi$ is a pure scalar field. Insertion of (2) into (1) leads to the following form of the Stueckelberg-modified Lagrangian density $\left[\mathcal{L}_{(S)}\right]$ in any arbitrary dimension of spacetime

$$
\mathcal{L}_{(P)} \longrightarrow \mathcal{L}_{(S)}=-\frac{1}{4} F_{\mu \nu} F^{\mu \nu}+\frac{m^{2}}{2} A_{\mu} A^{\mu} \mp m A_{\mu} \partial^{\mu} \phi+\frac{1}{2} \partial_{\mu} \phi \partial^{\mu} \phi,
$$

which respects [i.e. $\delta_{g} \mathcal{L}_{(S)}=0$ ] the following continuous and infinitesimal local classical gauge symmetry transformations $\left(\delta_{g}\right)$

$$
\delta_{g} A_{\mu}=\partial_{\mu} \chi, \quad \delta_{g} \phi= \pm m \chi, \quad \delta_{g} F_{\mu \nu}=0,
$$

where $\chi(x)$ is the local gauge transformation parameter $*$. The latter can be exploited within the purview of BRST formalism to derive the off-shell nilpotent (anti-)BRST symmetry transformations $\left[s_{(a) b}\right]$ in terms of the fermionic (anti-)ghost fields $(\bar{C}) C$ as (see, e.g. [7])

$$
\begin{aligned}
& s_{a b} A_{\mu}=\partial_{\mu} \bar{C}, \quad s_{a b} \bar{C}=0, \quad s_{a b} C=-i B, \quad s_{a b} B=0, \quad s_{a b} \phi= \pm m \bar{C}, \\
& s_{b} A_{\mu}=\partial_{\mu} C, \quad s_{b} C=0, \quad s_{b} \bar{C}=i B, \quad s_{b} B=0, \quad s_{b} \phi= \pm m C,
\end{aligned}
$$

${ }^{*}$ The Lagrangian density (3) is endowed with the first-class constraints: $\Pi^{0} \approx 0, \vec{\nabla} \cdot \vec{E} \mp m \Pi_{\phi} \approx 0$ where $\Pi^{0}=-F^{00} \approx 0$ is the primary constraint and $\frac{\partial \Pi^{0}}{\partial t}=\vec{\nabla} \cdot \vec{E} \mp m \Pi_{\phi} \approx 0$ is the secondary constraint with $\Pi_{\phi}=\dot{\phi} \mp m A_{0}$ as the canonical conjugate momentum w.r.t. the $\phi$ field. These first-class constraints are present in the generator $G=\int d^{D-1} x\left[\dot{\chi} \Pi^{0}-\chi\left(\vec{\nabla} \cdot \vec{E} \mp m \Pi_{\phi}\right)\right]$ which leads to the derivation of the local and infinitesimal classical gauge symmetry transformations (4) in any arbitrary dimension of spacetime. 
which are respected by the following (anti-)BRST invariant Lagrangian density $\left(\mathcal{L}_{b}\right)$ for the modified Proca theory in any arbitrary dimension of spacetime, namely,

$$
\begin{aligned}
\mathcal{L}_{b} & =\mathcal{L}_{(S)}+s_{b} s_{a b}\left[\frac{i}{2} A^{\mu} A_{\mu}-\frac{i}{2} \phi^{2}-\frac{1}{2} \bar{C} C\right] \\
& \equiv \mathcal{L}_{(S)}+s_{b}\left[-i \bar{C}\left\{(\partial \cdot A) \pm m \phi+\frac{1}{2} B\right\}\right] \\
& \equiv \mathcal{L}_{(S)}+s_{a b}\left[i C\left\{(\partial \cdot A) \pm m \phi+\frac{1}{2} B\right\}\right]
\end{aligned}
$$

where $B$ is the Nakanishi-Lautrup auxiliary field and the fermionic $\left(C^{2}=0, \bar{C}^{2}=0, C \bar{C}+\right.$ $\bar{C} C=0$ ) (anti-)ghost fields $(\bar{C}) C$ are invoked to maintain the unitarity in the theory. In its full blaze of glory, the Lagrangian density $\mathcal{L}_{b}$ looks as:

$$
\begin{aligned}
\mathcal{L}_{b} & =-\frac{1}{4} F_{\mu \nu} F^{\mu \nu}+\frac{m^{2}}{2} A_{\mu} A^{\mu} \mp m A_{\mu} \partial^{\mu} \phi+\frac{1}{2} \partial_{\mu} \phi \partial^{\mu} \phi \\
& +B(\partial \cdot A \pm m \phi)+\frac{B^{2}}{2}-i \partial_{\mu} \bar{C} \partial^{\mu} C+i m^{2} \bar{C} C
\end{aligned}
$$

It is straightforward to check that $s_{b} \mathcal{L}_{b}=\partial_{\mu}\left[B \partial^{\mu} C\right]$ and $s_{a b} \mathcal{L}_{b}=\partial_{\mu}\left[B \partial^{\mu} \bar{C}\right]$ which render the $D$-dimensional action integral $S=\int d^{D} x \mathcal{L}_{b}$ invariant under $s_{(a) b}$.

According to the celebrated Noether theorem, the above continuous, off-shell nilpotent $\left(s_{(a) b}^{2}=0\right)$, absolutely anticommuting $\left(s_{b} s_{a b}+s_{a b} s_{b}=0\right)$ and infinitesimal symmetry transformations lead to the following explicit expressions for the (anti-)BRST invariant $\left(s_{a b} J_{(a b)}^{\mu}=0, s_{b} J_{(b)}^{\mu}=0\right)$ Noether conserved currents $\left(J_{(r)}^{\mu}\right.$ with $\left.r=a b, b\right)$ :

$$
\begin{aligned}
J_{(a b)}^{\mu} & =-F^{\mu \nu} \partial_{\nu} \bar{C}+B \partial^{\mu} \bar{C} \pm m \bar{C} \partial^{\mu} \phi-m^{2} \bar{C} A^{\mu} \\
J_{(b)}^{\mu} & =-F^{\mu \nu} \partial_{\nu} C+B \partial^{\mu} C \pm m C \partial^{\mu} \phi-m^{2} C A^{\mu} .
\end{aligned}
$$

The conservation law $\partial_{\mu} J_{(r)}^{\mu}=0$ (with $r=b, a b$ ) can be proven by using the following Euler-Lagrange (EL) equations of motion (EoM), namely,

$$
\begin{aligned}
& \partial_{\mu} F^{\mu \nu}=\partial^{\nu} B \pm m \partial^{\nu} \phi-m^{2} A^{\nu}, \quad\left(\square+m^{2}\right) C=0, \\
& \square \phi \mp m(\partial \cdot A)= \pm m B, \quad\left(\square+m^{2}\right) \bar{C}=0 .
\end{aligned}
$$

The above conserved currents lead to the definition of conserved charges as

$$
\begin{aligned}
Q_{b}=\int d^{D-1} x J_{(b)}^{0} & =\int d^{D-1} x\left[-F^{0 i}\left(\partial_{i} C\right)+B \dot{C} \pm m C \dot{\phi}-m^{2} A_{0} C\right] \\
& \equiv \int d^{D-1} x[B \dot{C}-\dot{B} C] \\
Q_{a b}=\int d^{D-1} x J_{(a b)}^{0} & =\int d^{D-1} x\left[-F^{0 i}\left(\partial_{i} \bar{C}\right)+B \dot{\bar{C}} \pm m \bar{C} \dot{\phi}-m^{2} A_{0} \bar{C}\right] \\
& \equiv \int d^{D-1} x[B \dot{\bar{C}}-\dot{B} \bar{C}],
\end{aligned}
$$

where we have applied the Gauss divergence theorem to drop the total space derivative terms and used: $\dot{B}=\vec{\nabla} \cdot \vec{E}+m^{2} A_{0} \mp m \dot{\phi}$ [that arises from Eq. (9)] to derive the concise 
forms of conserved $\oplus^{\dagger}$ charges $Q_{(a) b}$. It is straightforward to note that the following exact forms of the charges w.r.t. $s_{(a) b}$ are true, namely,

$$
\begin{aligned}
& Q_{b}=\int d^{D-1} x\left[s_{b}\{i \dot{\bar{C}} C-i \bar{C} \dot{C}\}\right] \equiv \int d^{D-1} x\left[s_{a b}(i C \dot{C})\right], \\
& Q_{a b}=\int d^{D-1} x\left[s_{a b}\{i \bar{C} \dot{C}-i \dot{\bar{C}} C\}\right] \equiv \int d^{D-1} x\left[s_{b}(-i \bar{C} \dot{\bar{C}})\right],
\end{aligned}
$$

which establish the off-shell nilpotency and absolute anticommutativity of the conserved (anti-)BRST charges as illustrated below

$$
\begin{aligned}
& s_{b} Q_{b}=-i\left\{Q_{b}, Q_{b}\right\}=0 \quad \Longrightarrow \quad Q_{b}^{2}=0 \quad \Leftrightarrow \quad s_{b}^{2}=0, \\
& s_{a b} Q_{a b}=-i\left\{Q_{a b}, Q_{a b}\right\}=0 \quad \Longrightarrow \quad Q_{a b}^{2}=0 \quad \Leftrightarrow \quad s_{a b}^{2}=0, \\
& s_{a b} Q_{b}=-i\left\{Q_{b}, Q_{a b}\right\}=0 \quad \Longrightarrow \quad s_{a b}^{2}=0 \quad \Leftrightarrow \quad\left\{Q_{b}, Q_{a b}\right\}=0, \\
& s_{b} Q_{a b}=-i\left\{Q_{a b}, Q_{b}\right\}=0 \quad \Longrightarrow \quad s_{b}^{2}=0 \quad \Leftrightarrow \quad\left\{Q_{a b}, Q_{b}\right\}=0,
\end{aligned}
$$

where the basic principle behind the continuous symmetry transformations and their generators has been used. It is interesting to point out that the off-shell nilpotency [i.e. $Q_{(a) b}^{2}=0$ ] of the conserved (anti-)BRST charges $\left(Q_{(a) b}\right)$ is deeply related with the off-shell nilpotency [i.e. $s_{(a) b}^{2}=0$ ] of the (anti-)BRST transformations $s_{(a) b}$. However, we note that the absolute anticommutativity of the BRST charge with the anti-BRST charge is connected with the nilpotency $\left(s_{a b}^{2}=0\right)$ of the anti-BRST transformations $\left(s_{a b}\right)$. On the other hand, the absolute anticommutativity of the anti-BRST charge with the BRST charge is intemately connected with the off-shell nilpotency $\left(s_{b}^{2}=0\right)$ of the BRST transformations $\left(s_{b}\right)$. These statements are corroborated by a close and careful look at Eqs. (11) and (12). These observations will be very useful when we shall discuss the conserved (anti-)co-BRST charges and corresponding symmetries for the Stückelberg-modified 2D Proca theory in the fourth section.

\section{Stückelberg Formalism for the 2D Proca Theory: Mathematical Basis for Its Modification}

The 2D Proca theory is very special in the sense that the field strength tensor $F_{\mu \nu}$ has only one existing component which is nothing but the electric field (i.e. $F_{01}=E$ ). The latter (i.e. the electric field E) turns out to be a pseudo-scalar because it is a single object which changes sign under the parity symmetry transformation. Furthermore, as far as the mathematical aspect of the Stückelberg modification [cf. Eq. (2)] in any arbitrary dimension of spacetime is concerned, we note that the vector field $A_{\mu}$ (belonging to the 1-form $\left.A^{(1)}=d x^{\mu} A_{\mu}\right)$ is modified by a 1 -form $\Phi^{(1)}=d \Phi^{(0)}=d \phi \equiv d x^{\mu} \partial_{\mu} \phi$ where the 0 -form $\Phi^{(0)}=\phi$ is a pure scalar field and $d=d x^{\mu} \partial_{\mu}$ is the exterior derivative of the differential geometry [8-10]. A close look at Eq. (2) shows that the 0-form scalar field $\phi$ has

†The conservation law (i.e. $\left.\dot{Q}_{(a) b}=0\right)$ for the concise forms of the (anti-)BRST charges $Q_{(a) b}$ can be easily proven by using the EL-EoMs: $\left(\square+m^{2}\right) B=0,\left(\square+m^{2}\right) C=0,\left(\square+m^{2}\right) \bar{C}=0$ which emerge out from the variation of the action integral $S=\int d^{D-1} x \mathcal{L}_{b}$ defined w.r.t. the Lagrangian density $\mathcal{L}_{b}$. 
been incorporated into the Stückelberg modification with a mass factor in the denominator on the dimensional ground (in the natural units: $\hbar=c=1$ ). It is straightforward to note that, for the 2D Proca theory, the mass dimensions of $A_{\mu}$ and $\phi$ fields are zero. Hence, the modification in Eq. (2) is correct on the dimensional ground. As a passing remarks, it is interesting to point out that the field strength tensor $F_{\mu \nu}$ remains invariant under the modification (2) due to the well-known Stückelberg formalism.

As pointed out earlier, the two $(1+1)$-dimensional $(2 \mathrm{D})$ theory is very special because we have the freedom to add/subtract a pseudo-scalar field $\tilde{\phi}$ in the modification (2) with some condition. In other words, an axial-vector 1-form $\tilde{\Phi}^{(1)}=d \tilde{\Phi}^{(0)} \equiv d x^{\mu} \partial_{\mu} \tilde{\phi}$ constructed with a pseudo-scalar field $\tilde{\phi}$ (i.e. the 0 -form $\tilde{\Phi}^{(0)}=\tilde{\phi}$ ) is at our disposal. However, the 1 -form $\tilde{\Phi}^{(1)}$ is a pseudo-vector (i.e. axial-vector). To make it a polar-vector in $2 \mathrm{D}$, we have to take a single Hodge duality $*$ operation on it (with the input: $\varepsilon^{\mu \nu}=-\varepsilon^{\nu \mu}$ ), as follows:

$$
* \tilde{\Phi}^{(1)}=* d x^{\mu} \partial_{\mu} \tilde{\phi}=\varepsilon^{\mu \nu} d x_{\nu} \partial_{\mu} \tilde{\phi} \equiv d x^{\mu}\left(-\varepsilon_{\mu \nu} \partial^{\nu} \tilde{\phi}\right)
$$

where, at this stage, the explicit expression: $-\varepsilon_{\mu \nu} \partial^{\nu} \tilde{\phi}$ is a polar-vector in $2 \mathrm{D}$ modified version of Proca theory. In the mathematical language, the modification (2) can now be expressed, in terms of the 1-forms, as

$$
A^{(1)} \longrightarrow A^{(1)} \mp \frac{1}{m} d \Phi^{(0)} \pm \frac{1}{m} * d \tilde{\Phi}^{(0)}
$$

which leads to the following explicit modification of the Stückelberg technique, namely,

$$
A_{\mu} \longrightarrow A_{\mu} \mp \frac{1}{m} \partial_{\mu} \phi \mp \frac{1}{m} \varepsilon_{\mu \nu} \partial^{\nu} \tilde{\phi} .
$$

It should be pointed out that the mass term $(m)$ has to be present in the third term, too, in the above equation on the dimensional ground because the mass dimension of the pseudoscalar field $(\tilde{\phi})$ is also zero in $2 \mathrm{D}$ theory. As a side remark, we note that the field-strength tenor $F_{\mu \nu}$ does not remain invariant under (15) [which was invariant under the original modification (2)]. In fact, the field-strength tensor $F_{\mu \nu}$ explicitly transforms, under the modified Stückelberg-technique (15), as follows:

$$
F_{\mu \nu} \longrightarrow F_{\mu \nu} \mp \frac{1}{m}\left[\varepsilon_{\nu \lambda} \partial_{\mu} \partial^{\lambda} \tilde{\phi}-\varepsilon_{\mu \lambda} \partial_{\nu} \partial^{\lambda} \tilde{\phi}\right] .
$$

The special feature of $2 \mathrm{D}$ theory is the observation that only $F_{01}=E$ exists for the field strength tensor $F_{\mu \nu}$. This implies that the following is true due to (16), namely,

$$
F_{01} \longrightarrow F_{01} \mp\left(\partial_{1} \partial_{1} \tilde{\phi}-\partial_{0} \partial_{0} \tilde{\phi}\right) \text {. }
$$

In other words, the electric field $\left(E=F_{01}\right)$ of the Proca theory is modified, under the modified 2D Stückelberg-technique (15) (with the choice: $\varepsilon_{01}=+1=\varepsilon^{10}$ ), as follows:

$$
E \longrightarrow E \pm \frac{1}{m} \square \tilde{\phi}, \quad \square=\partial_{0} \partial_{0}-\partial_{1} \partial_{1}
$$


Thus, ultimately, the 2D Proca Lagrangian density $\left(\mathcal{L}_{(P)}^{(2 D)}\right)$ is modified to $\mathcal{L}_{(S)}^{(2 D)}$

$$
\begin{aligned}
\mathcal{L}_{(P)}^{(2 D)}=\frac{1}{2} E^{2}+\frac{m^{2}}{2} A_{\mu} A^{\mu} & \longrightarrow \\
& \mathcal{L}_{(S)}^{(2 D)}=\frac{1}{2}\left(E \pm \frac{1}{m} \square \tilde{\phi}\right)^{2} \pm m E \tilde{\phi}-\frac{1}{2} \partial_{\mu} \tilde{\phi} \partial^{\mu} \tilde{\phi} \\
& +\frac{m^{2}}{2} A_{\mu} A^{\mu} \mp m A_{\mu} \partial^{\mu} \phi+\frac{1}{2} \partial_{\mu} \phi \partial^{\mu} \phi,
\end{aligned}
$$

where $\mathcal{L}_{(S)}^{(2 D)}$ is the 2D Stückelberg-modified version of the Lagrangian density for the Proca theory (modulo some total spacetime derivative terms). We lay emphasis on the fact that the square of the first-term of (19) leads to

$$
\left(E \pm \frac{1}{m} \square \tilde{\phi}\right)^{2}=E^{2}+\frac{1}{m^{2}}(\square \tilde{\phi})^{2} \pm \frac{2}{m} E \square \tilde{\phi}
$$

where the last two terms are the higher derivative terms for a $2 \mathrm{D}$ theory in view of the fact that $E=-\varepsilon^{\mu \nu} \partial_{\mu} A_{\nu}$ (with the inputs: $\varepsilon^{\mu \nu}=-\varepsilon^{\nu \mu}, \varepsilon^{10}=+1=\varepsilon_{01}$ ) also contains a partial derivative. However, there is a solution to this problem if we assume, at this stage, that the pseudo-scalar field $\tilde{\phi}$ obeys the Klein-Gordon equation $f:\left(\square+m^{2}\right) \tilde{\phi}=0$. Thus, ultimately, the substitution $\square \tilde{\phi}=-m^{2} \tilde{\phi}$ leads to the following form of the Lagrangian density from the modified Lagrangian density in Eq. (19), namely,

$$
\mathcal{L}_{(S)}^{(2 D)}=\frac{1}{2}(E \mp m \tilde{\phi})^{2} \pm m E \tilde{\phi}-\frac{1}{2} \partial_{\mu} \tilde{\phi} \partial^{\mu} \tilde{\phi}+\frac{m^{2}}{2} A_{\mu} A^{\mu} \mp m A_{\mu} \partial^{\mu} \phi+\frac{1}{2} \partial_{\mu} \phi \partial^{\mu} \phi
$$

which has been considered by us in our earlier works $[6,7]$. However, this is for the firsttime, we are able to derive the Lagrangian density (21) by exploiting the theoretical beauty and strength of the 2D modified Stückelberg technique that has been quoted in Eq. (15).

At this crucial juncture, there are a few remarks on the theoretical beauty of the Lagrangian density (21). First of all, we find that the kinetic terms for the pure scalar field $(\phi)$ and pseudo-scalar field $(\tilde{\phi})$ carry opposite signs. In other words, the pseudo-scalar field $(\tilde{\phi})$ carries a negative kinetic term which is interesting from the point of view of the existence of the possible candidates for the dark matter and dark energy (see. e.g. [11, 12]). Furthermore, such fields (which have been christened as the "ghost" and "phantom" fields in the realm of modern-day cosmology) play important roles in the cyclic, bouncing and self-accelerated cosmological models of the Universe (see. e.g. [13-15]). Second, the signs of all the terms of the Lagrangian density (21), along with the gauge-fixing term [3] in the 't Hooft gauge $\left(\mathcal{L}_{g f}\right)[\mathrm{cf} . \mathrm{Eq} .(7)]$, namely,

$$
\begin{aligned}
\mathcal{L}_{(S)}^{(2 D)}+\mathcal{L}_{(g f)} & =\frac{1}{2}(E \mp m \tilde{\phi})^{2} \pm m E \tilde{\phi}-\frac{1}{2} \partial_{\mu} \tilde{\phi} \partial^{\mu} \tilde{\phi}+\frac{m^{2}}{2} A_{\mu} A^{\mu} \\
& \mp m A_{\mu} \partial^{\mu} \phi+\frac{1}{2} \partial_{\mu} \phi \partial^{\mu} \phi-\frac{1}{2}(\partial \cdot A \pm m \phi)^{2}
\end{aligned}
$$

\footnotetext{
${ }^{\ddagger}$ We shall see later that the final Lagrangian density [cf. Eqs. (22), (27)], with the replacement: $\square \tilde{\phi}=-m^{2} \tilde{\phi}$, does lead to the derivation of the Klein-Gordon equation [i.e. $\left.\left(\square+m^{2}\right) \tilde{\phi}=0\right]$ for the pseudo-scalar field [cf. Eqs. (22) and (24) below] which has been used in the derivation of (21) from (19).
} 
are fixed as it respects a set of a couple of very useful discrete duality symmetry transformations (modulo some total spacetime derivative terms). These duality transformations for the basic fields $\left(A_{\mu}, \phi, \tilde{\phi}\right)$ along with $E$ and $(\partial \cdot A)$ are listed as follows:

$$
\begin{array}{ll}
A_{\mu} \rightarrow \mp i \varepsilon_{\mu \nu} A^{\nu}, & \phi \rightarrow \mp i \tilde{\phi}, \quad \tilde{\phi} \rightarrow \mp i \phi, \\
E \rightarrow \pm i(\partial \cdot A), & (\partial \cdot A) \rightarrow \pm i E .
\end{array}
$$

Third, we note that the Lagrangian density (22) leads to the EL-EoMs for the fields $\phi$ and $\tilde{\phi}$ as the Klein-Gordon equations, namely,

$$
\left(\square+m^{2}\right) \phi=0, \quad\left(\square+m^{2}\right) \tilde{\phi}=0 .
$$

It is interesting to point out that one of the above equations [i.e. $\left.\left(\square+m^{2}\right) \tilde{\phi}=0\right]$ has been used in the derivation of (22) from (19). Fourth, we note that the mass term for the Proca field $A_{\mu}$ (i.e. $\frac{m^{2}}{2} A_{\mu} A^{\mu}$ ) remains invariant under the discrete duality symmetry transformations (23). As a consequence, the fields $\phi$ and $\tilde{\phi}$ carry the same rest mass $(m)$. Fifth, we note that the 2D modified Stückelberg-technique (15) remains invariant under the discrete duality symmetry transformations (23). Finally, we observe that the gauge-fixed Lagrangian density (22) respects the local and infinitesimal gauge and dual-gauge symmetry transformations $\delta_{g}$ and $\delta_{d g}$, respectively, as listed below (see, e.g. [7])

$$
\begin{aligned}
& \delta_{g} A_{\mu}=\partial_{\mu} \Sigma, \quad \delta_{g} \phi= \pm m \Sigma, \quad \delta_{g} E=0, \quad \delta_{g} \tilde{\phi}=0, \\
& \delta_{d g} A_{\mu}=-\varepsilon_{\mu \nu} \partial^{\nu} \Omega, \quad \delta_{d g} \tilde{\phi}=\mp m \Omega, \quad \delta_{d g} E=\square \Omega, \quad \delta_{d g} \phi=0,
\end{aligned}
$$

if we impose exactly the same kinds of restrictions $\left(\square+m^{2}\right) \Sigma=0,\left(\square+m^{2}\right) \Omega=0$ on the gauge and dual-gauge symmetry transformation parameters $\Sigma$ and $\Omega$ because we note that the following transformations of the Lagrangian density (22) are true, namely,

$$
\begin{aligned}
\delta_{g}\left[\mathcal{L}_{(S)}^{(2 D)}+\mathcal{L}_{(g f)}\right] & =-(\partial \cdot A \pm m \phi)\left[\square+m^{2}\right] \Sigma \\
\delta_{d g}\left[\mathcal{L}_{(S)}^{(2 D)}+\mathcal{L}_{(g f)}\right] & =\partial_{\mu}\left[m \varepsilon^{\mu \nu}\left(m A_{\nu} \Omega \pm \phi \partial_{\nu} \Omega\right) \pm m \tilde{\phi} \partial^{\mu} \Omega\right] \\
& +(E \mp m \tilde{\phi})\left[\square+m^{2}\right] \Omega .
\end{aligned}
$$

We shall see in the next section that, within the framework of BRST formalism, there is no outside restrictions on the (anti-)ghost fields which are the generalization of the classical gauge and dual-gauge transformation parameters $\Sigma$ and $\Omega$ to the quantum level.

\section{Comments on (Anti-)BRST and (Anti-)Co-BRST Symmetries: 2D Modified Proca Theory}

The (anti-)BRST and (anti-)co-BRST symmetry invariant Lagrangian density for the 2D modified Proca theory is the generalization of the (anti-)BRST invariant Lagrangian density

\footnotetext{
$\S$ The root-cause behind (23) is the self-duality condition on $2 \mathrm{D}$ Abelian 1-form. In other words, we note that: $* A^{(1)}=*\left(d x^{\mu} A_{\mu}\right)=d x^{\mu}\left(-\varepsilon_{\mu \nu} A^{\nu}\right) \equiv d x^{\mu} \tilde{A}_{\mu}$. The transformation $A_{\mu} \rightarrow \mp i \varepsilon_{\mu \nu} A^{\nu}$ in $(23)$ owes its origin to the self-duality condition where the dual-vector $\tilde{A}_{\mu}=-\varepsilon_{\mu \nu} A^{\nu}$.

$\mathbb{I}$ Invariance of the modified Stückelberg-technique (15) under the discrete symmetry transformations (23) and/or (29) demonstrates that there is a prefect duality symmetry in the 2D modified version of Proca theory. In fact, we note that (15) and (23) are intertwined together in a subtle fashion.
} 
(7) (valid in any arbitrary D-dimensions of spacetime) a: [l] (see, e.g. [7])

$$
\begin{aligned}
\mathcal{L}_{(B)} & =\mathcal{B}(E \mp m \tilde{\phi})-\frac{1}{2} \mathcal{B}^{2} \pm m E \tilde{\phi}-\frac{1}{2} \partial_{\mu} \tilde{\phi} \partial^{\mu} \tilde{\phi}+\frac{m^{2}}{2} A_{\mu} A^{\mu}+\frac{1}{2} \partial_{\mu} \phi \partial^{\mu} \phi \\
& \mp m A_{\mu} \partial^{\mu} \phi+B(\partial \cdot A \pm m \phi)+\frac{1}{2} B^{2}-i \partial_{\mu} \bar{C} \partial^{\mu} C+i m^{2} \bar{C} C,
\end{aligned}
$$

where we have $(i)$ invoked Nakanishi-Lautrup auxiliary field $\mathcal{B}$ to linearize the kinetic term of the Lagrangian density (22), and (ii) chosen a specific form of the Lagrangian density. In fact, there are four Lagrangian densities corresponding to (22) which have been discussed in our earlier work [7]. We have taken only one specific form in (27) which is different from the choices taken in [7]. It is straightforward to note that the (anti-)BRST transformations (5) are now generalized with additional transformations for our 2D theory as:

$$
\begin{array}{lccc}
s_{a b} A_{\mu}=\partial_{\mu} \bar{C}, & s_{a b} \bar{C}=0, & s_{a b} C=-i B, & s_{a b} \phi= \pm m \bar{C}, \\
s_{a b} B=0, & s_{a b} E=0, & s_{a b} \mathcal{B}=0, & s_{a b} \tilde{\phi}=0, \\
s_{b} A_{\mu}=\partial_{\mu} C, & s_{b} C=0, & s_{b} \bar{C}=+i B, & s_{b} \phi= \pm m C, \\
s_{b} B=0, & s_{b} E=0, & s_{b} \mathcal{B}=0, & s_{b} \tilde{\phi}=0 .
\end{array}
$$

Once again, we note that $s_{b} \mathcal{L}_{B}=\partial_{\mu}\left[B \partial^{\mu} C\right], s_{a b} \mathcal{L}_{B}=\partial_{\mu}\left[B \partial^{\mu} \bar{C}\right]$. Hence, the action integral $S=\int d^{2} x \mathcal{L}_{(B)}$ remains (anti-)BRST invariant for the physical fields which vanishoff as $x \rightarrow \pm \infty$ due to the application of Gauss's divergence theorem.

Before we derive the (anti-)co-BRST symmetry transformations from (28), it is interesting to point out that the discrete duality symmetry transformations (23) are generalized for the (anti-)BRST and (anti-)co-BRST invariant 2D Lagrangian density $\mathcal{L}_{(B)}$ as:

$$
\begin{array}{ll}
A_{\mu} \rightarrow \mp i \varepsilon_{\mu \nu} A^{\nu}, & \phi \rightarrow \mp i \tilde{\phi}, \quad \tilde{\phi} \rightarrow \mp i \phi, \quad B \rightarrow \mp i \mathcal{B}, \quad \mathcal{B} \rightarrow \mp i B, \\
C \rightarrow \pm i \bar{C} & \bar{C} \rightarrow \pm i C, \quad(\partial \cdot A) \rightarrow \pm i E \quad E \rightarrow \pm i(\partial \cdot A) .
\end{array}
$$

In other words, we find that the Lagrangian density $\mathcal{L}_{(B)}$ remains invariant under (29). As a consequence of the discrete duality symmetry transformations (29), we find that the nilpotent (anti-)co-BRST symmetry transformations $\left[s_{(a) d}\right]$ can be derived from the nilpotent (anti-)BRST symmetry transformations (28) as follows:

$$
\begin{array}{lrrr}
s_{a d} A_{\mu}=-\varepsilon_{\mu \nu} \partial^{\nu} C, \quad s_{a d} C=0, & s_{a d} \bar{C}=+i \mathcal{B}, & s_{a d} \mathcal{B}=0, & s_{a d} \phi=0, \\
s_{a d} B=0, & s_{a d} \tilde{\phi}=\mp m C, & s_{a d}(\partial \cdot A)=0, & s_{a d} E=\square C, \\
s_{d} A_{\mu}=-\varepsilon_{\mu \nu} \partial^{\nu} \bar{C}, \quad s_{d} \bar{C}=0, & s_{d} C=-i \mathcal{B}, & s_{d} \mathcal{B}=0, & s_{d} \phi=0, \\
s_{d} B=0, & s_{d} \tilde{\phi}=\mp m \bar{C}, & s_{d}(\partial \cdot A)=0, & s_{d} E=\square \bar{C} .
\end{array}
$$

Let us dwell a bit on the derivation of (30) from (28) and vice-versa by exploiting the sheer beauty of the discrete duality symmetry transformations (29). Let us first focus on the gauge field $A_{\mu}$ which transforms under the (anti-)BRST symmetry transformations as: $s_{a b} A_{\mu}=\partial_{\mu} \bar{C}, s_{b} A_{\mu}=\partial_{\mu} C$. From these nilpotent transformations, we can derive

\footnotetext{
${ }^{\|}$We would like to point out that our choice of the 2D Lagrangian density in Eq. (27) is quite different from the thread-bare discussions on various forms of the Lagrangian densities in our earlier work [7].
} 
the off-shell nilpotent (i.e. fermionic) (anti-)co-BRST symmetry transformations $s_{a d} A_{\mu}=$ $-\varepsilon_{\mu \nu} \partial^{\nu} C, s_{d} A_{\mu}=-\varepsilon_{\mu \nu} \partial^{\nu} \bar{C}$ by using the transformations (29) and the replacements $s_{b} \rightarrow s_{d}, s_{a b} \rightarrow s_{a d}$. It can be explicitly checked that $s_{b} A_{\mu}=\partial_{\mu} C$ goes to $s_{d}\left(\mp i \varepsilon_{\mu \nu} A^{\nu}\right)=$ $\partial_{\mu}( \pm i \bar{C})$ under $(29)$ which, ultimately, implies that $s_{d} A_{\mu}=-\varepsilon_{\mu \nu} \partial^{\nu} \bar{C}$. In exactly similar fashion, we note that $s_{a b} A_{\mu}=\partial_{\mu} C$ goes to $s_{a d}\left(\mp i \varepsilon_{\mu \nu} A^{\nu}\right)=\partial_{\mu}( \pm i C)$ under (29) and the replacement $s_{a b} \rightarrow s_{a d}$, which finally, leads to $s_{a d} A_{\mu}=-\varepsilon_{\mu \nu} \partial^{\nu} C$. We can repeat this exercise with $s_{b} \bar{C}=i B, s_{a b} C=-i B$ which, due to (29) and replacements $s_{b} \rightarrow s_{d}, s_{a b} \rightarrow$ $s_{a d}$, lead to the derivation of $s_{d} C=-i \mathcal{B}, s_{a d} \bar{C}=+i \mathcal{B}$. We lay emphasis on the fact that the reciprocal relationships also exist**. To be precise, we can obtain $s_{b} A_{\mu}=\partial_{\mu} C$ from the co-BRST symmetry transformation $s_{d} A_{\mu}=-\varepsilon_{\mu \nu} \partial^{\nu} \bar{C}$ by the replacement $s_{d} \rightarrow s_{b}$ and the discrete duality transformations (29). In other words, we have: $s_{b}\left(\mp i \varepsilon_{\mu \nu} A^{\nu}\right)=$ $-\varepsilon_{\mu \nu} \partial^{\nu}( \pm i C)$ from $s_{d} A_{\mu}=-\varepsilon_{\mu \nu} \partial^{\nu} \bar{C}$ which implies $s_{b} A_{\mu}=\partial_{\mu} C$. Thus, we can derive (28) from (30), too. In exactly similar manner, we observe that the concise forms of the (anti-)co-BRST charges $Q_{(a) d}$, for our present 2D theory, can be derived from the concise forms of the (anti-)BRST charges (10), for $D=2$, as

$$
Q_{d}=\int d x[\mathcal{B} \dot{\bar{C}}-\dot{\mathcal{B}} \bar{C}], \quad Q_{a d}=\int d x[\mathcal{B} \dot{C}-\dot{\mathcal{B}} C]
$$

due to the discrete symmetry transformations (29). Hence, the nilpotent symmetry transformations (28) and (30) as well as the conserved charges (10) and (31) are interconnected due to the duality symmetry transformations (29). To be more precise, in the mathematical language, we have a set of very interesting mappings: $s_{(a) b} \leftrightarrow s_{(a) d}$ and $Q_{(a) b} \leftrightarrow Q_{(a) d}$ due to the existence of a couple of very beautiful duality symmetry transformations (29) in our theory. It is an elementary exercise to note that we can also derive the concise forms of $Q_{b}$ and $Q_{a b}$ for our 2D theory from $Q_{d}$ and $Q_{a d}$ [cf. Eq. (31)] by using the discrete duality symmetry transformations (29).

We end this section with a couple of final remarks. First, the (anti-)co-BRST symmetry transformations (30), derived from the (anti-)BRST symmetry transformations (28), are the symmetry transformations for the Lagrangian density (27) as evident from the following:

$$
\begin{aligned}
& s_{a d} \mathcal{L}_{(B)}=\partial_{\mu}\left[(\mathcal{B} \pm m \tilde{\phi}) \partial^{\mu} C \pm m \varepsilon^{\mu \nu}\left(\phi \partial_{\nu} C \mp m C A_{\nu}\right)\right] \\
& s_{d} \mathcal{L}_{(B)}=\partial_{\mu}\left[(\mathcal{B} \pm m \tilde{\phi}) \partial^{\mu} \bar{C} \pm m \varepsilon^{\mu \nu}\left(\phi \partial_{\nu} \bar{C} \pm m \bar{C} A_{\nu}\right)\right]
\end{aligned}
$$

As a consequence of the the above observations, it is clear that the action integral $S=$ $\int d^{2} x \mathcal{L}_{(B)}$ remains invariant under (30) for the physical fields which vanish-off as $x \rightarrow$ $\pm \infty$ due to Gauss's divergence theorem. Second, we note that all the exact forms of the (anti-)BRST charges [that have been expressed in Eq. (11)] can be replicated in the context of conserved and nilpotent (anti-)co-BRST charges. The latter can be expressed in terms of the nilpotent (anti-)co-BRST symmetry transformations due to the replacements $Q_{b} \rightarrow Q_{d}, Q_{a b} \rightarrow Q_{a d}, s_{b} \rightarrow s_{d}, s_{a b} \rightarrow s_{a d}$ along with the discrete duality symmetry transformations that have been listed in Eq. (29).

${ }^{* *}$ It is worthwhile to mention that, in an earlier work (see, for e.g. [7]), the derivation of $s_{d} A_{\mu}=-\varepsilon_{\mu \nu} \partial^{\nu} \bar{C}$ from $s_{b} A_{\mu}=\partial_{\mu} C$ has been carried out for the on-shell nilpotent $s_{d}$ and $s_{b}$. However, the reciprocal relationship has not been established in [7].

${ }^{\dagger \dagger}$ Though the duality symmetry transformations (29) are able to provide the relationships between the 


\section{Conclusions}

One of the main highlights and key results of our present investigation is the modification in the Stückelberg-technique [cf. Eq. (15)] for our 2D massive Abelian 1-form gauge theory which has led to the derivation of the Lagrangian density (21) that is the fulcrum of our whole discussion in our present investigation. For our 2D modified version of Proca theory (i.e. a massive Abelian 1-form theory) the most fundamental symmetries are the continuous (anti-)BRST and (anti-)co-BRST transformations along with the discrete duality transformations (29). From these fermionic continuous symmetries, one can define a unique bosonic continuous symmetry transformation. There is a continuous ghost-scale symmetry, too, in our theory. An elegant blend of continuous and discrete symmetry transformations provide the physical realizations (see, e.g. [6, 7] for details) of the de Rham cohomological operators of the differential geometry [8-10] . The discrete duality symmetry transformations [cf. Eq. (29)], in particular, provide the physical realization of the Hodge duality $*$ operation of the differential geometry $[6,7]$ where it has been established that $s_{(a) d}= \pm * s_{(a) b} *$. This relationship provides the analogue of the mathematical relationship $\delta= \pm * d *$ of differential geometry that exists between the co-exterior derivative $(\delta)$ and the exterior derivative $(d)$. In our present investigation, we have shown the direct utility of the duality transformations (29) in establishing the mapping $s_{(a) d} \leftrightarrow s_{(a) b}$ which has not been accomplished in [7] for the off-shell nilpotent (anti-)BRST $\left(s_{(a) b}\right)$ and (anti-)co-BRST symmetry transformations $\left(s_{(a) d}\right)$. Furthermore, the Lagrangian density (27) is different from the Lagrangian density considered in [7]. The physical state of our theory is the harmonic state (in the Hodge decomposed state) that is annihilated by the (anti-)BRST and (anti-)co-BRST charges which lead to the annihilation of the physical state (out of total states of the quantum Hilbert space) by the operator forms of the firstclass constraints and their dual versions. For instance, it is evident that $Q_{(a) b} \mid$ phys $>=0$ implies that $B \mid$ phys $>=0$ and $\dot{B} \mid$ phys $>=0$. In other words, the first-class constraints $B\left(=\Pi^{0}\right) \mid$ phys $>=0$ and $\dot{B}\left(=\vec{\nabla} \cdot \vec{E} \mp m \Pi_{\phi}\right) \mid$ phys $>=0$ annihilate the physical states which are consistent with the Dirac quantization condition $\neq$. In exactly similar fashion, the condition: $Q_{(a) d} \mid$ phys $>=0$ leads to the $\mathcal{B} \mid$ phys $>=0$ and $\dot{\mathcal{B}} \mid$ phys $>=0$ which imply that $(E \mp m \tilde{\phi}) \mid$ phys $>=0$ and $\frac{d}{d t}(E \mp m \tilde{\phi}) \mid$ phys $>=0$ which are nothing but the dual versions of the first-class constraints [as can be seen by applying (29) on the first-class constraints]. These conditions are very sacrosanct and these are valid at any arbitrary loop-level diagrams in perturbation theory. In our very recent work [18], we have been able to show the importance of the annihilation of the physical state by the dual versions of the first-class constraints which, ultimately, imply that the 2D ABJ anomaly term (i.e. the

continuous symmetry transformations $s_{(a) b}$ and $s_{(a) d}$ and corresponding conserved charges, these continuous symmetries and conserved charges have their own independent identity as do the exterior and co-exterior derivatives of differential geometry [8-10].

${ }^{\ddagger \ddagger}$ It can be also seen that the conditions: $B \mid$ phys $>=0$ and $\dot{B} \mid$ phys $>=0$ (which emerge out from the physicality criteria: $Q_{(a) b} \mid$ phys $\left.>=0\right)$ imply that: $(\partial \cdot A \pm m \phi) \mid$ phys $>=0$ and $\frac{d}{d t}(\partial \cdot A \pm m \phi) \mid p h y s>=0$ where $(\partial \cdot A \pm m \phi)$ is the dual of the quantity $(E \mp m \tilde{\phi})$. The latter restriction [i.e. $(E \mp m \tilde{\phi}) \mid p h y s>=0]$ on the physical state emerges out from $Q_{(a) d} \mid$ phys $>=0$. The discussion on the various aspects of constraints, gauge symmetry transformations, symmetry generators, etc., has been clarified very beautifully in a couple of earlier works (see, e.g. $[16,17])$. 
pseudo-scalar electric field E) is trivial in an interacting 2D gauge theory where there is a coupling between the gauge field and matter (Dirac) fields. In fact, we have proved that our 2D interacting Proca theory (with the Dirac fields) is also a tractable field-theoretic example of Hodge theory (see, e.g. [18] for details). Our present discussion can be generalized for the massive 4D Abelian 2-form and 6D Abelian 3-form gauge theories where there will be modifications of the celebrated Stückelberg formalism because the above higher $p$-form $(p=2,3 \ldots)$ gauge theories have been proven to be the tractable examples of Hodge theory $[19,20]$. It will be a nice future endeavor to discuss the Stückelberg-modified SUSY QED where the question of infrared problem and the existence of the ultralight particles as the dark matter candidates have been discussed in a very nice piece of recent work [21].

\section{Acknowledgments}

One of us (AKR) gratefully acknowledges the financial support from the BHU-fellowship program of the Banaras Hindu University (BHU), Varanasi, under which the present investigation has been carried out. The authors express their deep sense of gratitude to the esteemed anonymous Reviewer for very sharp comments and useful suggestions.

Data availability statement: No new data were created or analysed in this study.

\section{References}

[1] DIRAC P. A. M., Lectures on Quantum Mechanics (Belfer Graduate School of Science, Yeshiva University Press, New York) 1964.

[2] SUNDERMEYER K., Constrained Dynamics: Lect. Notes Phys, Vol. 169, Springer-Verlag, Berlin 1982.

[3] RUEGG H. and RUIZ-AltaBA M., Int. J. Mod. Phys. A, 19 (2004) 3347.

[4] KUMAR R. and KRISHNA S., Eur. Phys. J. C, 77 (2017) 387.

[5] KRISHnA S., KUMAR R. and MALIK R. P., Ann. Phys., 414 (2020) 168087.

[6] BHAnjA T., SHUKLA D. and MALIK R. P., Eur. Phys. J. C, 73 (2013) 2535.

[7] CHAUHAN B., KUMAR S., TRIPATHI A. and MALIK R. P., Adv. High Energy Phys., 2020 (2020) 3495168.

[8] EGUCHI T., GILKEY P. B. and HANSON A., Physics Reports, 66 (1980) 213.

[9] MUKHI S. and MUKANDA N., Introduction to Topology, Differential Geometry and Group Theory for Physicists, Wiley Eastern Private Limited, New Delhi, 1990.

[10] van HOLTON J. W., Phys. Rev. Lett., 64 (1990) 2863. 
[11] ZHURAVLEV V. M., KORNILOV D. A. and SAVELOVA E. P., Gen. Relativ. and Gravit., 36 (2004) 1736.

[12] AHARONOV Y., POPESCU S., ROHRLICH D. and VAIDMAN L., Physical Review A, 48 (1993) 4084.

[13] STEINHARDT P. J. and TUROK N., Science, 296 (2002) 1436.

[14] NOVELlO M. and BeRGLiAfFA S. E. P., Physics Reports, 463 (2008) 127.

[15] KOYAMA K., Class. Quantum Grav., 24 (2007) R231.

[16] Mitra P. and RAJARAMAn R., Ann. Phys., 203 (1990) 137.

[17] MITRA P. and RAJARAMAN R., Ann. Phys., 203 (1990) 157.

[18] TRIPATHI A., RAO A. K. and MALIK R. P., arXiv: 2105.10960 [hep-th].

[19] GUPTA S. and MALIK R. P., Eur. Phys. J. C, 58 (2008) 517.

[20] KUMAR R., KRISHNA S., SHUKLA A. and MALIK R. P., Int. J. Mod. Phys. A, 29 (2014) 1450135.

[21] VINZE R., GOVINDARAJAN T. R., MISRA A. and RAMADEVI P., Mod. Phys. Lett. A, 35 (2020) 2050303. 